\title{
INFLUÊNCIA DAS CONDIÇÕES DO PROCESSO DE SECAGEM NA QUALIDADE DE FATIAS DE PEQUI (Caryocar brasiliense Camb.)
}

\author{
K. S. MENDONÇA ${ }^{1 *}$, F. V. FIGUEIRA ${ }^{1}$, M. A. CIRILLO ${ }^{2}$, J. R. J. JUNQUEIRA ${ }^{1}$, C.A. BORGO ${ }^{3}$, \\ J. L. G. CORRÊA ${ }^{1}$ \\ ${ }^{1}$ Universidade Federal de Lavras, Departamento de Ciência dos Alimentos \\ ${ }^{2}$ Universidade Federal de Lavras, Departamento de Ciência Exatas \\ ${ }^{3}$ FIEC - Fundação Indaiatubana de Educação e Cultura \\ *e-mail: keamendonca@msn.com
}

\begin{abstract}
RESUMO
O pequi é uma fruta do cerrado brasileiro que contém altos teores de ácido ascórbico (AA) e carotenóides. No entanto, o consumo de pequi ainda é limitado devido à alta perecibilidade do fruto. O objetivo deste trabalho foi estabelecer as melhores condições para a produção de fatias secas de polpa de pequi, estudando diferentes mecanismos de secagem (convecção ou vácuo, $40{ }^{\circ} \mathrm{C}$ e $60{ }^{\circ} \mathrm{C}$ ) precedidos ou não por pré-tratamento osmótico (40\% e $60 \%$ de concentração de solução de sacarose). Foi observado que os pré-tratamentos de desidratação osmótica diminuíram a duração do processo de secagem e a variação de volume do produto seco. No entanto, promoveram lixiviação de componentes funcionais, como AA e carotenóides. $\mathrm{O}$ emprego de vácuo e baixa temperatura de secagem, sem pré-tratamentos osmóticos, foram superiores a outras condições de secagem, porque produziram fatias de polpa de pequi secas com altos teores de AA e carotenóides e coeficiente de reidratação, moderada duração do processo de secagem e pequenas alterações no volume e na coloração das amostras.
\end{abstract}

\section{INTRODUÇÃO}

Pequi (Caryocar brasiliense Camb.) é uma fruta típica do cerrado brasileiro, com altos níveis de carotenóides e ácido ascórbico. A sua exportação é limitada pela falta de técnicas apropriadas de conservação (MACHADO; MELLO; HUBINGER 2013).

A secagem é uma técnica que confere estabilidade e prolonga a vida útil dos alimentos. O pequi seco pode ser utilizado como ingrediente em preparações domésticas e industriais, enriquecendo os pratos com moléculas funcionais, além de potencializar a exploração comercial desse fruto. No entanto, os processos de secagem devem levar em conta a preservação nutricional do pequi.

A secagem convectiva é um processo muito empregado comercialmente. Mas, devido à longa duração, está associado à redução da qualidade sensorial e nutricional dos produtos (MUJUMDAR; LAW 2010). Em geral, o pré-tratamento osmótico melhora a qualidade de produtos secos, inibindo o escurecimento enzimático e retendo a cor natural do produto (CORRÊA et al. 2011). Reduz também o encolhimento e o endurecimento (OIKONOMOPOULOU; KROKIDA 2013) e melhora as propriedades sensoriais e nutricionais do produto seco (LOMBARD et al. 2008). O uso de vácuo durante a secagem evita a oxidação de vitaminas e antioxidantes (ORIKASA et al. 2014). O ácido ascórbico e os carotenóides são moléculas termossensíveis e facilmente oxidáveis durante a secagem em atmosfera normal (GÜMÜŞAY et al. 2015). 
O objetivo deste trabalho foi estabelecer as melhores condições para a produção de fatias secas de polpa de pequi através da avaliação de secagens por convecção e a vácuo, precedidas ou não por pré-tratamento osmótico.

\section{MATERIAL E MÉTODOS}

Amostras de pequi (Caryocar brasiliense Camb.) foram colhidas na região de cerrado do norte de Minas Gerais, Brasil. O mesocarpo externo foi removido manualmente e descartado. As amostras foram preparadas com fatias do mesocarpo interno $(35,04 \pm 1,77 \times 14,61 \pm 2,12 \times 3,42 \pm 0,73$ $\mathrm{mm})$. As amostras frescas foram armazenadas em sacos plásticos opacos sob refrigeração a $18 \pm 2^{\circ} \mathrm{C}$ até a execução dos experimentos. $\mathrm{O}$ descongelamento das amostras foi feito sob refrigeração a $4^{\circ} \mathrm{C}$ por $2 \mathrm{~h}$ e depois à temperatura ambiente até atingir o equilíbrio térmico com o ambiente.

Os ensaios de secagem seguiram um delineamento experimental fatorial completo $3 \times 2 \times 2$ considerando a concentração da solução osmótica ( $40 \%$ e $60 \%$ de sacarose), a temperatura de secagem $\left(40^{\circ} \mathrm{C}\right.$ e $\left.60^{\circ} \mathrm{C}\right)$ e o tipo de secagem (convectiva e sob pressão de vácuo de $10 \mathrm{kPa}$ ). Foram realizados doze tratamentos, codificados conforme a Tabela 1

As soluções osmóticas foram preparada com água destilada e sacarose comercial, nas concentrações de $40 \%$ e $60 \%$ $(\mathrm{p} / \mathrm{p})$. Os tratamentos osmóticos foram realizados durante 120 minutos a $25^{\circ} \mathrm{C}$ em câmara termoestática (Eletrolab EL111/4, São Paulo, Brasil). A razão entre solução:amostra foi mantida a 10:1 (p:p) (VIANA; CORRÊA; JUSTUS 2014) para evitar a diluição da solução durante o processo osmótico. Os experimentos foram realizados em triplicata e a média, relatada nos resultados.

Finalizado o processo osmótico, as fatias de pequi foram removidas das soluções e imediatamente imersas em banho de água e gelo durante $10 \mathrm{~s}$ a fim de parar a desidratação e remover a solução osmótica restante na superfície. Em seguida, foram gentilmente drenadas com papel absorvente (CORRÊA et al. 2014).

Tabela 1 - Delineamento experimental.

\begin{tabular}{lccc}
\hline Tratamento & $\begin{array}{c}\text { Concentração da } \\
\text { solução osmótica } \\
{[\%]}\end{array}$ & $\mathrm{T}\left[{ }^{\circ} \mathrm{C}\right]$ & $\begin{array}{c}\text { Tipo de } \\
\text { secagem }\end{array}$ \\
\hline P40T40V & 40 & 40 & vácuo \\
P40T40C & 40 & 40 & convectiva \\
P40T60V & 40 & 60 & vácuo \\
P40T60C & 40 & 60 & convectiva \\
P60T40V & 60 & 40 & vácuo \\
P60T40C & 60 & 40 & convectiva \\
P60T60V & 60 & 60 & vácuo \\
P60T60C & 60 & 60 & convectiva \\
INT40V & - & 40 & vácuo \\
INT40C & - & 40 & convectiva \\
INT60V & - & 60 & vácuo \\
INT60C & - & 60 & convectiva \\
\hline
\end{tabular}

As fatias de pequi foram secas até o teor de umidade final de $12 \mathrm{~kg}$ água/100kg amostra. Os experimentos de secagem foram realizados nas condições previstas na Tabela 1, em estufa de escala laboratorial (Solab SL 104/40, Piracicaba, Brasil).

\subsection{Análises de Qualidade}

A atividade de água foi determinada a $25^{\circ} \mathrm{C}$ com auxílio de equipamento Aqualab (modelo 3TE, Decagon Devices Inc., Pullman, WA, USA) (YU; SCHMIDT; SCHMIDT 2009).

O teor de ácido ascórbico (AA) foi determinado com extração seguida de quantificação em HPLC. A extração das amostras foi realizada por adição de cerca de $1 \mathrm{~g}$ de amostra triturada e $5 \mathrm{~mL}$ de solução de $\mathrm{H}_{2} \mathrm{SO}_{4} \quad 0,05$ mol. $\mathrm{L}^{-1}$ e tratamento com ultrassom por $10 \mathrm{~min}$. Os extratos foram filtrados através de filtro $\mathrm{HV}$ Millex (Millipore), de polietileno, com porosidade de $0,45 \mu \mathrm{m}$. O volume de $20 \mu \mathrm{L}$ foi injetado na 
coluna cromatográfica de análise. A quantificação de ácido ascórbico (AA) foi realizada em sistema de HPLC (Shimadzu, Prominece, Japão) constituído por uma bomba de alta pressão (Shimadzu AD LC-20 modelo, Japão), amostrador automático (Shimadzu SIL-20A M, Japão) e um detector de arranjo de diodos (DAD) (Shimadzu SPDM20A, Japão). Foram utilizadas as seguintes condições cromatográficas: sistema de HPLC, DAD; coluna cromatográfica Shim-pack VP ODS (250 mm x 4,6 mm), coluna de guarda Shim-pack VP ODS $(5,0 \times 4,0 \mathrm{~mm})$, fase móvel e 0,005 mol.L ${ }^{-1}(\mathrm{pH}=3)$ de tampão de fosfato, com uma taxa de fluxo de $1,0 \mathrm{~mL}$ $\min ^{-1}$ a $25^{\circ} \mathrm{C}$, em método isocrático. Os cromatogramas foram obtidos a $254 \mathrm{~nm}$. A quantificação foi realizada usando o método do padrão externo.

A determinação de carotenóides totais foi realizada de acordo com a metodologia descrita por Rodrigues-Amaya (1999), com base na extração em acetona e separação em éter de petróleo. A determinação do teor de carotenóides totais foi feita em espectrôfotometro (Varian Cary 50 Probe, Austrália) por medição da absorbância a 450 nm ideal para $\beta$-caroteno, o carotenóide predominante em pequi.

A determinação da coloração da superfície das amostras foi feita pelo sistema CIELAB, $\left(\mathrm{L}^{*}, \mathrm{a}^{*}, \mathrm{~b}^{*}\right)$ lidas diretamente no colorímetro (Minolta CR400, Japão). A variação total de cor $(\triangle \mathrm{E})$ foi determinada através da Equação 1 (ORIKASA et al. 2014).

$$
\Delta \mathrm{E}=\sqrt{\left(\mathrm{L}^{*}-\mathrm{L}_{0}^{*}\right)^{2}+\left(\mathrm{a}^{*}-\mathrm{a}_{0}^{*}\right)^{2}+\left(\mathrm{b}^{*}-\mathrm{b}_{0}^{*}\right)^{2}}
$$

A variação de volume das amostras secas foi estimada usando o programa ImageJ 1.46r (National Institutes of Health, USA) para determinar a área superficial antes e após a secagem. As imagens foram capturadas com uma câmera digital (Sony DSC-WX50/B). A determinação do volume foi feita pela multiplicação da área pela espessura das amostras, obtidas com um paquímetro digital (Western, $150 \mathrm{~mm}$, precisão $\pm 0,01 \mathrm{~mm}$ ) (AKONOR; TORTOE 2014). As medidas foram feitas em triplicata. A extensão do encolhimento foi expressa em variação de volume (VV), quanto mais próximo da unidade, menor o encolhimento da amostra (OIKONOMOPOULOU; KROKIDA 2013) (Equação 2):

$$
\mathrm{VV}=\frac{\mathrm{V}^{\prime}}{\mathrm{V}_{0}^{\prime}}
$$

A capacidade de reidratação das amostras foi quantificada utilizando o coeficiente de reidratação ( $\left.\mathrm{R}_{\text {coef }}\right)$ (RAMALLO; MASCHERONI 2012) a $100^{\circ} \mathrm{C}$ (Equação 3):

$R_{\text {coef }}=\frac{m_{r h}\left(100-X_{0}\right)}{m_{d h}\left(100-X_{d h}\right)}$

\subsection{Análises Estatísticas}

Para determinar a semelhança entre os tratamentos de secagem foi usada uma análise de agrupamento hierárquico (dendograma). A probabilidade de significância para cada atributo na discriminação dos tratamentos foi avaliada por meio do teste $F$ ( $p$ valor $<0,05$ ).

Para identificar quais características das amostras secas são correlacionadas com os tratamentos, foram feitas análises estatísticas dessas correlações utilizando-se a técnica de biplots e análise de componentes principais (PCA) (JOLLIFFE 2002). Os dados para cada tratamento foram representados como a média das pontuações calculadas a partir de três repetições. A análise estatística foi realizada utilizando Chemoface 1.5 (UFLA, Lavras, Brasil). 


\section{RESULTADOS E DISCUSSÃO}

\subsection{Caracterização das Amostras Frescas}

As amostras frescas de fatias de polpa de pequi apresentaram teor de umidade de $51,49 \pm 1,64 \mathrm{~kg}$ de água $/ 100 \mathrm{~kg}$ de amostra; atividade de água de $0,986 \pm 0,002$; teor de

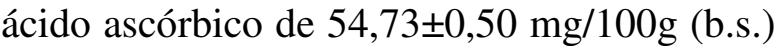
e de carotenóides de $121,02 \pm 11,35 \mathrm{mg} / \mathrm{g}$ (b.s.). Os resultados das análises de qualidade das amostras de fatias de polpa de pequi após a secagem em diferentes condições estão apresentados na Tabela 2.

Tabela 2 - Características de fatias de polpa de pequi após diversos tratamentos de secagem.

\begin{tabular}{|c|c|c|c|c|c|c|c|}
\hline Tratamentos & $a_{w}$ & VV & $\mathrm{R}_{\text {coef }}$ & $\begin{array}{c}\mathrm{t}_{\mathrm{sec}} \\
{[\mathrm{min}]}\end{array}$ & $\begin{array}{c}\text { AA } \\
{[\mathrm{mg} / 100 \mathrm{~g}]}\end{array}$ & $\begin{array}{l}\text { Carot. } \\
{[\mathrm{mg} / \mathrm{g}]}\end{array}$ & $\Delta \mathrm{E}$ \\
\hline & \multicolumn{7}{|c|}{ Amostras secas sem pré-tratamentos osmótico } \\
\hline INT40C & $0,438 \pm 0,004$ & $0,6163 \pm 0,0502$ & $0,9570 \pm 0,0135$ & 900 & $29,58 \pm 0,71$ & $53,37 \pm 6,22$ & $17,17 \pm, 25$ \\
\hline INT40V & $0,435 \pm 0,001$ & $0,7627 \pm 0,0454$ & $0,9851 \pm 0,0171$ & 840 & $43,65 \pm 0,35$ & $71,78 \pm 6,98$ & $10,23 \pm 2,44$ \\
\hline INT60C & $0,435 \pm 0,001$ & $0,5177 \pm 0,0413$ & $0,9034 \pm 0,0111$ & 780 & $21,64 \pm 0,14$ & $37,21 \pm 5,78$ & $14,92 \pm 3,16$ \\
\hline \multirow[t]{2}{*}{ INT60V } & $0,435 \pm 0,001$ & $0,6284 \pm 0,0378$ & $0,9749 \pm 0,0189$ & 780 & $36,48 \pm 0,45$ & $52,10 \pm 8,10$ & $14,35 \pm 1,70$ \\
\hline & \multicolumn{7}{|c|}{ Amostras secas pré-tratadas osmoticamente } \\
\hline P40T40C & $0,474 \pm 0,004$ & $0,7675 \pm 0,0545$ & $0,9595 \pm 0,0338$ & 360 & $16,78 \pm 0,97$ & $56,53 \pm 9,26$ & $8,18 \pm 3,37$ \\
\hline P40T40V & $0,471 \pm 0,001$ & $0,6623 \pm 0,0746$ & $0,8439 \pm 0,0010$ & 420 & $18,22 \pm 0,12$ & $73,70 \pm 10,93$ & $5,25 \pm 1,81$ \\
\hline P40T60C & $0,412 \pm 0,003$ & $0,8062 \pm 0,0235$ & $0,8378 \pm 0,0233$ & 120 & $16,14 \pm 0,43$ & $41,66 \pm 6,83$ & $13,60 \pm 3,41$ \\
\hline P40T60V & $0,408 \pm 0,003$ & $0,7671 \pm 0,0307$ & $0,9597 \pm 0,0240$ & 180 & $23,26 \pm 0,59$ & $55,54 \pm 9,10$ & $18,24 \pm 1,85$ \\
\hline P60T40C & $0,481 \pm 0,003$ & $0,8076 \pm 0,0247$ & $0,9050 \pm 0,0258$ & 120 & $16,00 \pm 0,78$ & $39,55 \pm 2,08$ & $10,71 \pm 2,11$ \\
\hline P60T40V & $0,472 \pm 0,001$ & $0,8626 \pm 0,0361$ & $0,8249 \pm 0,0259$ & 90 & $16,74 \pm 0,63$ & $52,73 \pm 2,77$ & $6,70 \pm 2,99$ \\
\hline Р60T60C & $0,427 \pm 0,003$ & $0,8493 \pm 0,0293$ & $0,7945 \pm 0,0169$ & 60 & $16,67 \pm 0,31$ & $29,14 \pm 1,53$ & $14,98 \pm 3,71$ \\
\hline P60T60V & $0,447 \pm 0,003$ & $0,8435 \pm 0,0451$ & $0,9179 \pm 0,0127$ & 90 & $31,03 \pm 0,45$ & $38,85 \pm 2,04$ & $16,48 \pm 1,60$ \\
\hline
\end{tabular}

Valores fornecidos como média seguidos pelo desvio padrão. Carot: teor de carotenóides (b.s.)

\subsection{Análise do Dendograma}

Considerando a análise do dendograma (Figura 1), o surgimento de três grupamentos permitiu a identificação do efeito do prétratamento osmótico na etapa de secagem, devido à separação do grupamento sem prétratamento (IN) dos demais.

Nas amostras pré-tratadas osmoticamente, foi possível observar o efeito da temperatura de secagem, pela separação das amostras de acordo com a temperatura de secagem (T40 e T60).

Baseado na discriminação destes grupamentos, foi realizado um teste $\mathrm{F}$ (Tabela
3) com o objetivo de detectar as variáveis significativas na formação dos grupos.

Figura 1 - Dendograma de similaridade

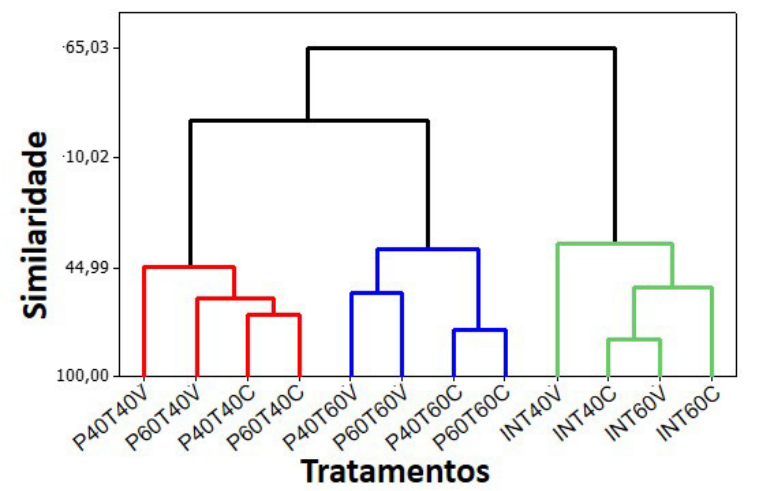


Tabela 3 - Análise da contribuição de cada variável na discriminação dos grupos de tratamentos

\begin{tabular}{cc}
\hline Variáveis resposta & p-valor \\
\hline $\mathrm{t}_{\mathrm{sec}}$ & $0,000^{*}$ \\
$\mathrm{a}_{\mathrm{W}}$ & $0,000^{*}$ \\
$\mathrm{VV}$ & $0,022^{*}$ \\
$\mathrm{R}_{\text {coef }}$ & 0,179 \\
Carotenóides & 0,297 \\
$\mathrm{AA}$ & $0,024^{*}$ \\
$\Delta \mathrm{E}$ & $0,003^{*}$ \\
\hline
\end{tabular}

"Indica significância estatística $(\mathrm{p}<0,05)$.

A significância do teste $\mathrm{F} \quad(\mathrm{p}<0,05)$ mostrou que $t_{s e c}, a_{w}, V V$, AA e $\Delta E$ foram as variáveis que estabeleceram a diferenciação entre os grupos. As amostras sem prétratamento (IN) apresentaram maior tempo de secagem $\left(t_{\mathrm{sec}}\right)$ e encolhimento após o processo de secagem. No entanto, o teor de AA foi maior para amostras sem pré-tratamento.

As alterações na estrutura do alimento pelo pré-tratamento osmótico levaram a redução do tempo de secagem $\left(t_{\text {sec }}\right)$. Além de remover parcialmente a água da matriz do produto por um método não-invasivo, contribuiu para um aumento da difusividade da água durante a secagem posterior (CORRÊA et al., 2011). Os fenômenos de encolhimento (VV) durante a secagem foram limitados nas amostras pré-tratadas osmoticamente (P40 e P60), devido à incorporação dos sólidos no pré-tratamento, que reforçaram a estrutura da célula de vegetal (GIOVANELLI; BRAMBILLA; SINELLI 2013; OIKONOMOPOULOU; KROKIDA 2013).

A principal desvantagem do prétratamento osmótico das fatias de pequi foi a perda de teor de AA. Em amostras prétratadas osmoticamente (P40 e P60), o teor de AA após o processo de secagem foi inferior, quando comparado com as amostras secas sem pré-tratamento (IN). Atribui-se esta perda a um processo de lixiviação dos compostos durante o processamento osmótico (DEVIC et al. 2010).

As amostras pré-tratadas e secas a $60^{\circ} \mathrm{C}$ (T60) apresentaram valores mais baixos de atividade de água $\left(\mathrm{a}_{\mathrm{w}}\right)$ (Tabela 2), devido à exposição à temperaturas mais elevadas. Foi observada maior eficiência do processo de remoção da água livre apesar de alcançar a mesma umidade final $(12 \mathrm{~kg}$ de água $/ 100 \mathrm{~kg}$ de amostra) que as amostras T40. No entanto, vale ressaltar que todos os níveis de atividade de água foram menores do que 0,600, portanto, capazes de assegurar a extensão da vida de prateleira do produto seco, minimizando alterações microbiológica, químicas e físicas (LEE; LIM 2011).

Apesar de mais efetiva na redução da atividade de água, as temperaturas superiores de secagem (T60) aumentaram a alteração de cor $(\Delta \mathrm{E})$ nas amostras secas pré-tratadas. Isso pode ter ocorrido devido à degradação de moléculas de carotenóides termossensíveis presentes nas fatias de polpa de pequi (RIBEIRO et al. 2014).

Foi aplicada uma análise de componentes principais para verificar quais tratamentos podem ser organizados em grupos, quais características contribuem para a formação dos grupos e estabelecer quais tratamentos são adequados para produzir fatias de pequi.

As Figuras 2A e 3A mostram os gráficos de scores das amostras sem prétratamento (IN) e com pré-tratamento osmótico (P40 e P60), respectivamente, representados no espaço definido pelos dois primeiros componentes principais. O biplot da intensidade dos atributos nos dois primeiros componentes principais são mostrados nas Figuras $2 \mathrm{~B}$ e $3 \mathrm{~B}$. Tratamentos que atribuem características semelhantes às amostras foram agrupados. Quanto maior a intensidade do atributo, maior a sua importância para cada agrupamento. Os dois primeiros componentes principais responsáveis por $95,08 \%$ e $76,11 \%$ da variabilidade total do sistema, no que diz 
respeito às amostras $\mathrm{IN}$ e pré-tratadas $(\mathrm{P} 40 \mathrm{e}$ P60), respectivamente.

Em amostras sem pré-tratamento (IN), Figura 2, em função da sua correlação com os atributos, o segundo componente principal permitiu a separação das amostras em dois grupos. O tratamento INT40C está na parte inferior (Grupo I), caracterizado por altos valores de atividade de água, tempo de secagem e $\Delta \mathrm{E}$, o que o diferenciou dos demais tratamentos que apresentaram atributos com intensidades opostas. A aplicação de temperatura por um longo período de tempo não é recomenda para secagem de alimentos porque pode afetar negativamente a qualidade de frutas secas e trazer degradação de componentes nutricionalmente importantes no alimento (YILMAZ et al. 2015). Para o outro grupo de amostras (Grupo II), o primeiro componente principal permitiu a separação das amostras em subgrupos, onde INT40V (subgrupo I) apresenta alto teor de AA, VV, carotenóides e coeficiente de reidratação, enquanto amostra INT60C (subgrupo III) apresentou valores baixos desses atributos e INT60V (subgrupo II) atributos de valor intermediário. Além disso, as amostras do Grupo II apresentaram menores tempo de secagem, alteração de cor e atividade de água.

As amostras de fatias de pequi secas sob vácuo e baixa temperatura, tal como utilizado no tratamento INT40V, mostraram ser as melhores entre os tratamentos testados. Com elevados teores de ácido ascórbico e carotenóides, alto coeficiente de reidratação, pequena variação de volume e de cor. $\mathrm{O}$ resultado positivo ocorreu porque, apesar da temperatura baixa, o uso do vácuo contribuiu para diminuir o tempo de secagem (BADWAIK et al. 2014). Além disso, o ácido ascórbico e os carotenóides são moléculas altamente sujeitas a reações oxidativas irreversíveis, de modo que a utilização de vácuo evitou a degradação desses compostos (ORIKASA et al. 2014; LIU; WU; MIAO 2014). Este efeito protetor foi aumentado com a utilização de temperaturas reduzidas, uma vez que estas moléculas são termossensíveis (GÜMÜŞAY et al. 2015; ORIKASA et al. 2014). Sogi, Siddiq e Dolan (2015) observaram que os teores de AA e de carotenóides em manga foram reduzidos no processo de secagem, devido aos danos causados pelo calor. Nas fatias de pequi secas isso pode ser verificado nos melhores resultados obtidos nas amostras tratadas sob vácuo (nas mesmas temperaturas) e pela diminuição gradual do teor de AA e carotenóides com o aumento da temperatura.

FIGURA 2 - Scores dos tratamentos (A) e biplots dos atributos (B) para amostras sem prétratamento (IN)
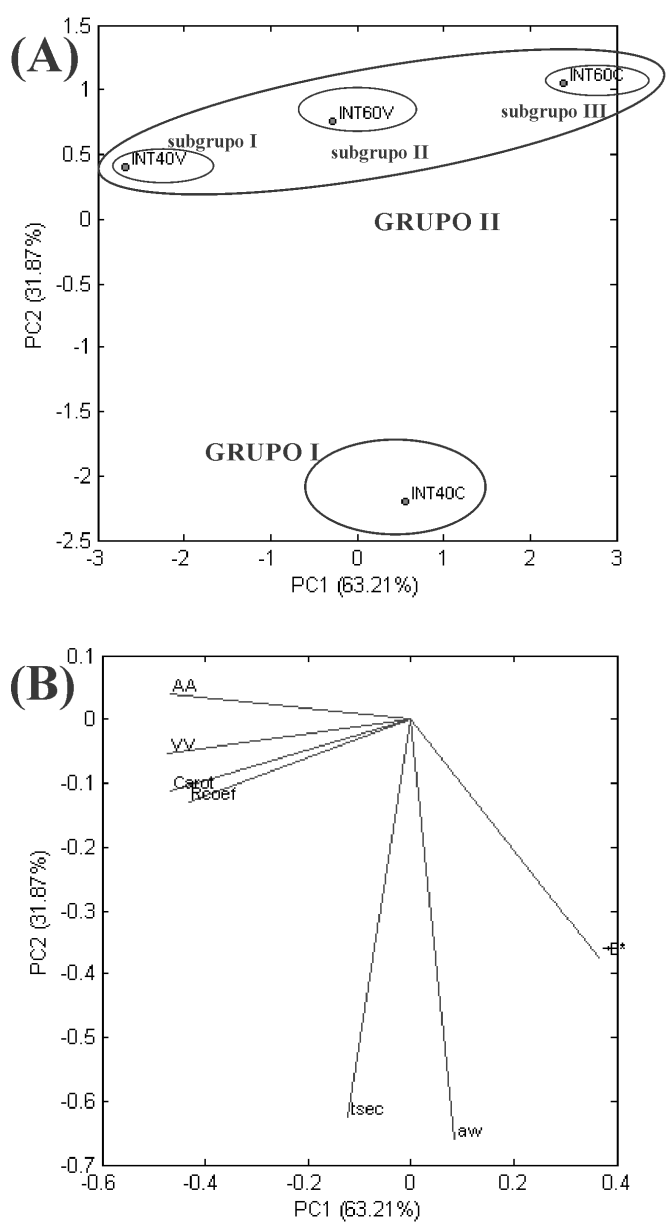
FIGURA 3 - Scores dos tratamentos (A) e biplots dos atributos (B) para amostras pré-tratadas osmoticamente (P40 e P60)
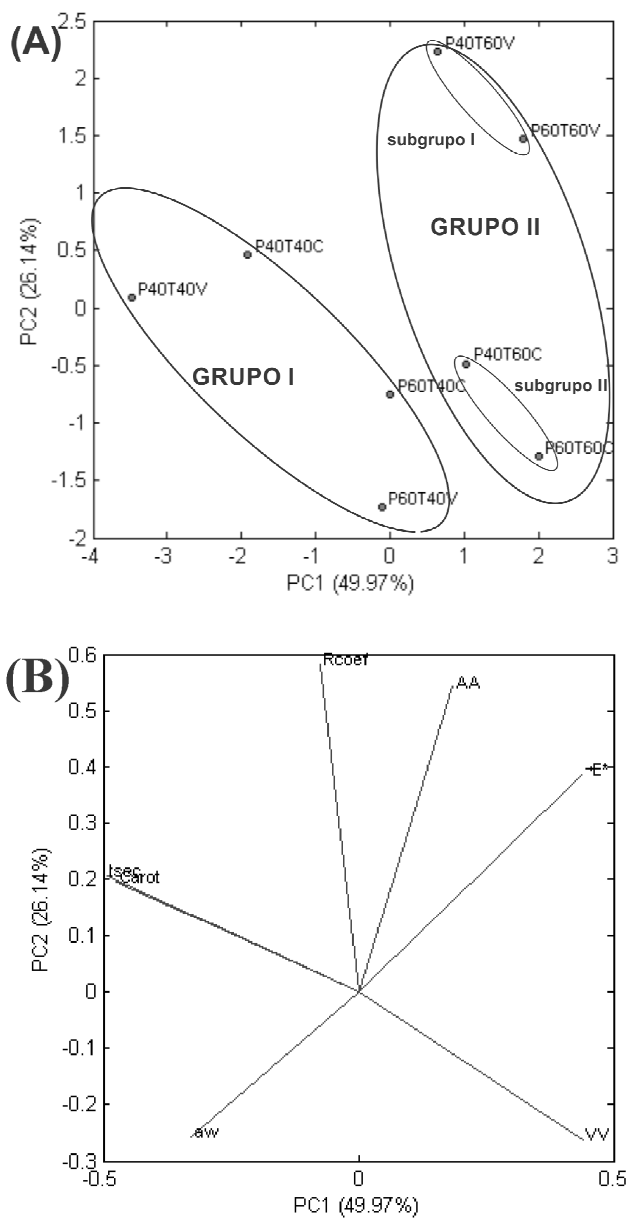

Variações no volume e o coeficiente de reidratação seguiram as tendências dos teores de AA e carotenóides, apresentando melhores valores para as amostras expostas ao vácuo e temperaturas baixas. Os maiores valores de coeficiente de reidratação pode ser atribuído principalmente à exposição à pressão reduzida durante a secagem. O uso de vácuo preservou a estrutura porosa desenvolvida durante $\mathrm{o}$ processo de secagem, promovendo maior coeficientes de reidratação na amostras (BADWAIK et al. 2014). A baixa temperatura também pode contribuir para o elevado coeficiente de reidratação, evitando o endurecimento que é observado em amostras sob temperaturas elevadas (PROTHON et al. 2001) e dificulta a reidratação.

A utilização de temperaturas baixas durante a secagem das fatias de pequi contribuiu para minimizar o colapso estrutural das amostras, para a redução das alterações de volume e para o aumento dos valores do coeficiente de reidratação. A temperatura durante o processo de secagem é o parâmetro mais importante que afeta a estrutura dos materiais secos (OIKONOMOPOULOU; KROKIDA 2013). As variações de volume (VV) durante a secagem e o coeficiente de reidratação $\left(\mathrm{R}_{\text {coef }}\right)$ estão relacionados às mudanças estruturais extensivas que ocorrem durante $o$ processo de secagem (OIKONOMOPOULOU; KROKIDA 2013). Quanto mais tênue do colapso estrutural, menos intensa a variação do volume e maior a capacidade de reidratação da amostra.

Os valores de atividade de água de todas as amostras secas foram inferiores a 0,481 . Este nível é suficiente para promover a estabilidade física, química e microbiológica em amostras secas $\left(a_{w}<0,60\right)$ (LEE; LIM 2011). Assim, por esse critério, todos os tratamentos de secagem foram satisfatórios.

Em amostras não pré-tratadas (IN), o tempo de secagem variou de 780 a 900 minutos e a variação de volume de 0,5177 a 0,7627 , estes atributos foram mais elevados do que os observados em amostras prétratadas osmoticamente (P40 e P60), cujos tempos de secagem variaram entre 420 e 60 min e VV entre 0,6623 e 0,8626 (Tabela 2). Por outro lado, o teor de AA foi substancialmente maior em amostras sem prétratamento, variando de 21,64 a 43,65 $\mathrm{mg} / 100 \mathrm{~g}$ e de 16,67 a $31,03 \mathrm{mg} / 100 \mathrm{~g}$ para amostras pré-tratadas. Assim, o efeito dos tratamentos deve ser devidamente avaliado levando em conta a qualidade do produto final.

Em amostras pré-tratadas osmoticamente (P40 e P60), o primeiro componente principal foi responsável pela 
diferenciação entre as amostras de acordo com a temperatura de secagem, tal como pode ser verificado nos scores (Figura 3A). Grupo I é formado por amostras T40 e Grupo II por amostras T60.

Devido ao uso de baixas temperaturas durante a secagem de fatias de pequi osmoticamente pré-tratadas, amostras do Grupo I apresentaram baixos valores $\Delta \mathrm{E}$ e alto teor de carotenóides. Estes atributos são benefícios conhecidos do emprego de temperaturas moderadas de secagem. Uma vez que os carotenóides são pigmentos termossensíveis, a sua preservação promove a minimização das alterações de cor (SANTANA et al. 2013; RIBEIRO et al. 2014). Por outro lado, o alongamento do tempo de secagem, que é esperado com o uso de baixas temperaturas, promoveu a degradação de AA e danos da estrutura da amostra, evidenciado pela maior variação de volume e baixo coeficiente de reidratação. Além disso, não houve qualquer efeito protetor do pré-tratamento osmótico sobre os compostos termossensíveis, na secagem (GIOVANELLI; BRAMBILLA; SINELLI 2013).

Em amostras submetidas à alta temperatura de secagem (T60), o segundo componente principal tornou possível dividir o grupo II em dois subgrupos (Figura 3A), nos quais a utilização de vácuo ou secagem convectiva pode distinguir os subgrupos. A utilização de temperatura elevada e secagem a vácuo (T60V, subgrupo I) promoveu a preservação do teor de AA e alto coeficiente de reidratação $\left(\mathrm{R}_{\mathrm{coef}}\right)$, além do processo de secagem rápida. Como características indesejáveis, o subgrupo I apresentou encolhimento, baixo teor de carotenóides e, consequentemente, variação de cor nas amostras secas. Tal como observado nas amostras sem pré-tratamento (IN), a utilização de vácuo também contribuiu para preservar o teor de AA em amostras pré-tratadas osmoticamente e secas a uma temperatura elevada, devido à restrição de oxigênio que inibe a decomposição de ácido ascórbico (ORIKASA et al. 2014). O uso de altas temperaturas em secagem convectiva (T60C, subgrupo II) não contribuiu para a qualidade das amostras secas, comprometendo atributos importantes. Produzindo amostras com baixos teores de ácido ascórbico e carotenóides, grande variação de cor e baixo coeficiente de reidratação.

\section{CONCLUSÃO}

Produzir fatias de polpa de pequi secas com a utilização de vácuo e baixas temperaturas, sem pré-tratamentos osmóticos, mostrou ser a melhor forma de obter produtos de qualidade. Este tratamento proporcionou altos valores de AA e carotenóides, coeficiente de reidratação e pequenas alterações de volume e de cor. A utilização do vácuo no secador contribuiu para reduzir o tempo de secagem, para preservar o teor de AA e de carotenóides e para o desenvolvimento de uma estrutura porosa durante o processo de secagem, promovendo melhor reidratação nas amostras. Este efeito na preservação da amostra foi reforçado pelo uso de baixas temperaturas, que também contribuíram para minimizar o colapso estrutural de fatias de pequi, diminuindo as variações de volume.

\section{NOMENCLATURA}

P40

P60 pré-tratamento com solução osmótica de $60 \%$

IN in natura

T40 secagem a $40^{\circ} \mathrm{C}$

T60 secagem a $60^{\circ} \mathrm{C}$

C secagem convectiva

$\mathrm{V}$ secagem a vácuo

$\mathrm{t}_{\mathrm{sec}} \quad$ tempo de secagem [min]

$\mathrm{a}_{\mathrm{w}} \quad$ atividade de água 


\begin{tabular}{|c|c|}
\hline VV & variação de volume \\
\hline $\mathrm{R}_{\text {coef }}$ & coeficiente de reidratação \\
\hline AA & $\begin{array}{l}\text { teor de ácido ascórbico } \\
{[\mathrm{mg} / 100 \mathrm{~g}]}\end{array}$ \\
\hline$\Delta \mathrm{E}$ & variação total de cor \\
\hline $\mathrm{V}^{\prime}$ & volume $\left[\mathrm{m}^{3}\right]$ \\
\hline $\mathrm{m}$ & massa da amostra (g) \\
\hline$X$ & $\begin{array}{l}\text { teor de umidade [kg água/ } 100 \mathrm{~kg} \\
\text { amostra] }\end{array}$ \\
\hline $\begin{array}{l}\text { b.s. } \\
\text { subscritos }\end{array}$ & base seca \\
\hline 0 & amostras frescas \\
\hline rh & amostras reidratadas \\
\hline dh & amostras desidratadas \\
\hline
\end{tabular}

\section{REFERÊNCIAS}

AKONOR, P.T.; TORTOE, C. Effect of Blanching and Osmotic Pre-treatment on Drying Kinetics, Shrinkage and Rehydration of Chayote (Sechium edule) during Convective Drying. British Journal of Applied Science and Technology, v.4, p.1215-1229, 2014.

BADWAIK, L.S.; CHOUDHURY, S.; BORAH, P.K.; SIT, N.; DEKA, S.C. Comparison of Kinetics and Other Related Properties of Bamboo Shoot Drying Pretreated with Osmotic Dehydration. Journal of Food Processing and Preservation, v.38, p.1171-1180, 2014.

CORRÊA, J.L.G.; DEV, S.R.S.; GARIEPY Y.; RAGHAVAN, G.S.V. Drying of pineapple by microwave-vacuum with osmotic pretreatment. Drying Technology, v. 29, p.1556-1561, 2011.

CORRÊA, J.L.G.; ERNESTO, D.B.; ALVES, J.G.L. F.; ANDRADE, R.S. Optimisation of vacuum pulse osmotic dehydration of blanched pumpkin. International Journal of Food Science \& Technology, v.49, p.20082014, 2014.
DEVIC, E.; GUYOT, S.; DAUDIN, J.D.; BONAZZI, C. Kinetics of polyphenol losses during soaking and drying of cider apples. Food and Bioprocess Technology, v.3, p.867-877, 2010.

GIOVANELLI, G.; BRAMBILLA, A.; SINELLI, N. Effects of osmo-air dehydration treatments on chemical, antioxidant and morphological characteristics of blueberries. LWT - Food Science and Technology, v.54, p.577-584, 2013.

GÜMÜŞAY, Ö.A.; BORAZAN, A.A.; ERCAL, N.; DEMIRKOL, O. Drying effects on the antioxidant properties of tomatoes and ginger. Food Chemistry, v.173, p.156-162, 2015 .

JOLLIFFE, I.T. Principal component analysis 2 nd., Berlin: Springer, 2002.

LEE, J.; LIM, L. Osmo-dehydration pretreatment for drying of pumpkin slice. International Food Research Journal, v.18, p.1223-1230, 2011.

LIU, Y.; WU, J.; MIAO, S. Effect of a Modified Atmosphere on Drying and Quality Characteristics of Carrots. Food Bioprocess Technology, v.7, p.2549-2559, 2014.

LOMBARD, G.E.; OLIVEIRA, J.C.; FITO, P.; ANDRÉS A. Osmotic dehydration of pineapple as a pre-treatment for further drying. Journal of Food Engineering, v.85, p.277-284, 2008.

MACHADO, M.T.C.; MELLO, B.C.B.S.; HUBINGER, M.D. Study of alcoholic and aqueous extraction of pequi (Caryocar brasiliense Camb.) natural antioxidants and extracts concentration by nanofiltration. Journal of Food Engineering, v.117, p.450457, 2013. 
MUJUMDAR, A.S.; LAW, C.L. Drying Technology: Trends and Applications in Postharvest Processing. Food Bioprocess Technology, v.3, p.843-852, 2010.

OIKONOMOPOULOU, V.P.; KROKIDA, M.K. Novel Aspects of Formation of Food Structure during Drying. Drying Technology, v.31, p.990-1007, 2013.

OLIVEIRA, M.E.B.; GUERRA, N.B.; MAIA, A.H.N.; ALVES, R.E; MATOS, N.M.S., SAMPAIO, F.G.M.; LOPES, M.M.T. Características químicas e físico-químicas de pequis da Chapada do Araripe, Ceará. Brasileira de Fruticultura, v.32, p.114-115, 2010.

ORIKASA, T.; KOIDE, S.; OKAMOTO, S.; IMAIZUMI, T.; MURAMATSU, Y., TAKEDA, J.; SHIINA, T., TAGAWAFET, A. Impacts of hot air and vacuum drying on the quality attributes of kiwifruit slices. Journal of Food Engineering, v.125, p.51$58,2014$.

PROTHON, F.; AHRNÉ, L.M.; FUNEBO, T.; KIDMAN, S.; LANGTON, M.; SJÖHOLM, I. Effects of combined osmotic and microwave dehydration of apple on texture, microstructure and rehydration characteristics. LWT - Food Science and Technology, v.34, p.95-101, 2001.

RAMALLO, L.; MASCHERONI, R.H. Quality evaluation of pineapple fruit during drying process. Food and Bioproducts Processing, v.90, p.275-283, 2012.

RIBEIRO, D.M.; FERNANDES, D.C.; ALVES, A.M.; NAVES, M.M.V. Carotenoids are related to the colour and lipid content of the pequi (Caryocar brasiliense Camb.) pulp from the Brazilian Savanna. Food Science and Technology, v.34, p.507-512, 2014.
RODRIGUES-AMAYA, D.B. Carotenoids and food preparation: the retention of provitamin A carotenoids in prepared, processed, and stored foods. Arlington: OMNI Project, 1999.

SANTANA, A.A.; KUROZAWA, L.E.; OLIVEIRA, R.A.; PARK, K.J. Influence of Process Conditions on the Physicochemical Properties of Pequi Powder Produced by Spray Drying. Drying Technology, v.31, p.825-836, 2013.

SOGI, D.S.; SIDDIQ, M; DOLAN, K.D. Total phenolics, carotenoids and antioxidant properties of Tommy Atkin mango cubes as affected by drying techniques. LWT - Food Science and Technology, v.62, p.1-5, 2015.

VIANA, A.D.; CORRÊA, J.L.G.; JUSTUS, A. Optimisation of the pulsed vacuum osmotic dehydration of cladodes of fodder palm. International Journal of Food Science \& Technology, v.49, p.726-732, 2014.

YILMAZ， F.M.; YÜKSEKKAYA， S.; VARDIN, H.; KARAASLAN, M. The effects of drying conditions on moisture transfer and quality of pomegranate fruit leather (pestil). Journal of the Saudi Society of Agricultural Sciences (in press), 2015.

YU, X.; SCHMIDT, A.R.; SCHMIDT, S.J. Uncertainty analysis of hygrometer-obtained water activity measurements of saturated salt slurries and food materials. Food Chemistry, v.115, p.214-226, 2009.

\section{AGRADECIMENTOS}

Os autores agradecem ao $\mathrm{CNPq}$, Fapemig e Capes, pelo apoio financeiro. 